\title{
Effect of low stressful temperature on genetic variation of five quantitative traits in Drosophila melanogaster
}

\author{
OA Bubliy ${ }^{1}$ and V Loeschcke ${ }^{2}$ \\ ${ }^{1}$ Vavilov Institute of General Genetics, Gubkin Street 3, 119991 Moscow, Russia; ${ }^{2}$ Department of Ecology and Genetics, University \\ of Aarhus, Ny Munkegade, Building 540, DK-8000 Aarhus C, Denmark
}

\begin{abstract}
A half-sib analysis was used to investigate genetic variation for three morphological traits (thorax length, wing length and sternopleural bristle number) and two life-history traits (developmental time and larva-to-adult viability) in Drosophila melanogaster reared at a standard $\left(25^{\circ} \mathrm{C}\right)$ and a low stressful $\left(13^{\circ} \mathrm{C}\right)$ temperature. Both phenotypic and environmental variation showed a significant increase under stressful conditions in all traits. For estimates of genetic variation, no statistically significant differences were found between the two environments. Narrow heritabilities tended to be higher at $13^{\circ} \mathrm{C}$ for sternopleural bristle number and viability and at $25^{\circ} \mathrm{C}$ for wing length and developmental time,
\end{abstract}

whereas thorax length did not show any trend. However, the pattern of genetic variances and evolvability indices (coefficient of genetic variation and evolvability), considered in the context of literature evidence, indicated the possibility of an increase in additive genetic variation for the morphological traits and viability and in nonadditive genetic variation for developmental time. The data suggest that the effect of stressful temperature may be trait-specific and this warns against generalizations about the behaviour of genetic variation under extreme conditions.

Heredity (2002) 89, 70-75. doi:10.1038/sj.hdy.6800104

Keywords: Drosophila melanogaster, environmental stress; genetic variation; heritability; low temperature

\section{Introduction}

The evolution of natural populations is known to depend on the presence of additive genetic variation in quantitative traits. Higher levels of this variation accelerate responses to natural selection and increase the probability for successful adaptation to environmental changes. Numerous quantitative genetic experiments indicate that the environment, by itself, can affect the amount of expressed additive variation. Under extreme conditions, estimates of narrow heritability and/or additive genetic variance tend to be higher than under optimal conditions and a hypothesis has been advanced that an enhanced level of additive genetic variation is favoured by environmental stress (Hoffmann and Parsons, 1991).

Quantitative variation in different environments has been extensively studied in the last decade using Drosophila (eg, Gebhardt and Stearns, 1992; David et al, 1994; Barker and Krebs, 1995; Hoffmann and Schiffer, 1998; Imasheva et al, 1998; Loeschcke et al, 1999; Bubliy et al, 2001). The results of these studies indicated the possibility of both higher and of lower additive genetic variances/heritabilities under stress as well as the absence of changes across environments. Most evidence for increased additive variation has been obtained in experiments (Barker and Krebs, 1995; de Moed et al, 1997;

Correspondence: OA Bubliy, Vavilov Institute of General Genetics, Gubkin Street 3, 119991 Moscow, Russia. E-mail: oleg_b@vigg.ru

Received 13 November 2001; accepted 3 April 2002
Imasheva et al, 1998, 1999, 2000; Karan et al, 1999; Loeschcke et al, 1999) in which genetic parameters have been estimated using the isofemale line technique, ie, a modified full-sib design with isofemale lines treated as families (Hoffmann and Parsons, 1988). However, the full-sib design is the least precise among all quantitative genetics methods developed to estimate additive genetic variance. Since the covariance among full sibs includes a relatively large proportion of the non-additive component of the genetic variance (Falconer and Mackay, 1996), estimates of the additive genetic variance from fullsib/isofemale line data may be biased.

In the present study, we consider the effects of environmental stress on genetic variation in D. melanogaster using a different experimental approach, the paternal half-sib design. The covariance among paternal half-sibs contains a smaller proportion of non-additive genetic variance than does the full-sib covariance (Falconer and Mackay, 1996) and thus provides more reliable estimates of the additive genetic variance. To stress flies, we reared them at $13^{\circ} \mathrm{C}$, which is close to the lower tolerance limit for $D$. melanogaster (David et al, 1983). Three morphological traits, thorax length, wing length and sternopleural bristle number, and two life-history traits, developmental time and larva-to-adult viability, were analysed. The effect of low rearing temperature on these traits has been examined recently in isofemale lines of $D$. melanogaster by de Moed et al (1997, thorax and wing length), Imasheva et al (1998, thorax and wing length, sternopleural bristle number, developmental time and viability; 2000, wing length), and Karan et al (1999, thorax and wing length). Also, Sgrò and Hoffmann (1998) compared variation for 
wing length and developmental time between different temperatures using parent-offspring regression, a method which gives estimates of the additive genetic variance similar to those for the half-sib design. In the isofemale line studies, a trend for an increase in additive genetic variation at low temperatures was reported for all the traits, whereas, in the parent-offspring comparisons, estimates of the additive genetic variance in the stressful environment decreased for developmental time and did not change for wing length.

\section{Materials and methods}

The laboratory D. melanogaster stock was founded from flies collected in Leiden (the Netherlands) in October 1999. Wild inseminated females were used to start 30 isofemale lines, which were maintained on a standard oatmealsugar-yeast-agar Drosophila medium for 30 generations (five generations at $25^{\circ} \mathrm{C}$ and then 25 generations at $20^{\circ} \mathrm{C}$ ). To obtain flies for the experiment, a mass population was established and maintained as discrete generations at $25^{\circ} \mathrm{C}$ with regular mixing of adults among bottles.

From the third generation of the mass population, a sample of 60 virgin males and 180 virgin females was taken and aged for 5-7 days. Further, each male (sire) was placed in an individual vial together with three females (dams). The vials contained the standard medium supplemented with live yeast to stimulate mating. Thirty-six hours later, each female was transferred for egg laying to a separate empty vial containing a plastic spoon, which was filled with $2 \mathrm{ml}$ of the yeast-seeded standard medium. After another $24 \mathrm{~h}$, the females were transferred to new vials with spoons to continue egg laying. First-instar larvae obtained from each female were transferred from spoons to six new vials (10 larvae per vial), three vials on the first day (block 1) and another three on the second day (block 2). All vials contained $7 \mathrm{ml}$ of the standard medium without live yeast. Immediately after transferring larvae, one set of the vials was placed at the stressful temperature and the other set at the control temperature. Each set consisted of 540 vials and represented 30 sire families. The two temperatures were $13 \pm 0.5^{\circ} \mathrm{C}$ and $25 \pm 0.5^{\circ} \mathrm{C}$, respectively.

Eclosed adults were collected and counted every $12 \mathrm{~h}$ until all flies had emerged. Females and males from each vial were pooled and stored in an individual plastic microtube filled with a fixative liquid ( $1 / 2$ glycerine $+1 / 2$ $70 \%$ ethanol). Three morphological traits, thorax length, left wing length and sternopleural bristle number on the left side of the fly, were measured on fixed females (one per vial). Thorax length (from the anterior margin of the thorax to the posterior tip of the scutellum from the dorsal view) and wing length (from the intersection of the wing margin and vein L3 to the intersection between this vein and the anterior crossvein) were measured in arbitrary micrometer units (70 units $=1 \mathrm{~mm}$ ) using an ocular micrometer and converted into millimeters. Developmental time was estimated as the time interval in days from the midpoint of the oviposition period to the recorded time of eclosion. For each vial, the mean developmental time (males and females pooled) was computed to be used as an individual observation in statistical analysis. Viability was calculated as the proportion of flies (sexes pooled) that emerged from each vial relative to the number of first-instar larvae used to set up the cultures. Thus, the number of observations for viability was also equal to the number of vials.

Data treatment was based on standard statistical procedures for the paternal half-sib analysis (Becker, 1992; Falconer and Mackay, 1996). The ANOVA design for three morphological traits at $13^{\circ} \mathrm{C}$ was slightly unbalanced due to the absence of females among emerged flies in some vials. Because of this, the significance of the mean squares (type III) was tested with Satterthwaite's approximation (Sokal and Rohlf, 1996). Viability data were analyzed as arcsine-square-root-transformed proportions; for other traits, no transformation was needed. Statistical comparisons for variance components and heritabilities were done with $t$-tests, using their approximate standard errors. In addition to these classical measures of genetic variability, coefficients of genetic variation and evolvabilities were calculated as $\mathrm{CV}_{\mathrm{A}}=100 \sqrt{ } V_{\mathrm{A}} / x$ and $I_{\mathrm{A}}=V_{\mathrm{A}} / x^{2}$, respectively, where $V_{\mathrm{A}}$ is the additive genetic variance and $x$ is the mean of a trait (Houle, 1992).

\section{Results}

Table 1 shows means, phenotypic variances and coefficients of variation for the five traits at 25 and $13^{\circ} \mathrm{C}$. There were highly significant differences between the two temperatures for all estimates $(P<0.001$; means, $t$-test; variances, F-ratio test; $\mathrm{CVs}$, Z-test). Rearing at $13^{\circ} \mathrm{C}$ resulted in decreased thorax length, bristle number and viability, whereas wing length and developmental time were higher at this temperature. Phenotypic variances and CVs were higher in the stressful environment and their increase was most pronounced for the life-history traits.

In Table 2, mean squares and variance components from three-way nested ANOVAs with sire, dam and block as random factors are presented. All morphological traits at both temperatures showed significant among-sire and among-dam variation and non-significant variation among experimental blocks. For developmental time, sire and dam effects were significant at 25 and $13^{\circ} \mathrm{C}$, respectively, while for viability variation among sires at $13^{\circ} \mathrm{C}$ was significant and variation among dams at $25^{\circ} \mathrm{C}$ marginally significant $(P=0.065)$. The effect of block was significant for the life-history traits in both environments, which might be due to unexpected technical problems with maintaining low temperature in the first day of the experiment.

Comparing among-sire and among-dam variances to test for nonadditive genetic effects $\left(s^{2}\right.$ dam $>s^{2}$ sire $)$ in particular traits did not reveal statistically significant differences (results not presented). Between-temperature comparisons of variance components on the among-sire, among-dam and among-block levels of variation showed differences $\left(13^{\circ} \mathrm{C}>25^{\circ} \mathrm{C}\right.$, two-tailed $\left.P<0.05\right)$ only for the among-dam variances in wing length and developmental time, but they could not be considered significant taking into account multiple comparisons (the Bonferroni correction). For the morphological traits, the values of $s^{2}$ sire and $s_{\text {dam }}^{2}$ as well as the sum $s^{2}$ sire $+s^{2}$ dam (full-sib variance) were generally higher at $13^{\circ} \mathrm{C}$ than at $25^{\circ} \mathrm{C}$. Similar trends were also evident for $s^{2}$ sire in viability, $s^{2}$ dam in developmental time and the sum $s^{2}$ sire $+s^{2}$ dam in both life-history traits. The among-progeny variance was significantly higher (two-tailed $P<0.001$ ) at $13^{\circ} \mathrm{C}$ for each trait, indicating an increase in environmental variation in the stressful environment. This increase was the most pronounced for developmental time and the least pronounced for sterno- 
Table 1 Means, phenotypic variances and coefficients of variation for five traits in D. melanogaster reared at two temperatures. The morphological traits are measured in females; the life-history traits are estimated using both females and males (pooled samples). For all traits at $25^{\circ} \mathrm{C}$ and for the life-history traits at $13^{\circ} \mathrm{C}$, the number of observations is 540; for the morphological traits at $13^{\circ}$ it is 531

\begin{tabular}{|c|c|c|c|c|c|c|}
\hline \multirow[t]{2}{*}{ Trait } & \multicolumn{3}{|r|}{$25^{\circ} \mathrm{C}$} & \multicolumn{3}{|r|}{$13^{\circ} \mathrm{C}$} \\
\hline & mean $\pm S E$ & $s^{2}$ & $C V \pm S E$ & mean $\pm S E$ & $s^{2}$ & $C V \pm S E$ \\
\hline Thorax length $(\mathrm{mm} \times 100)$ & $107.85 \pm 0.12$ & 8.04 & $2.63 \pm 0.08$ & $104.00 \pm 0.16$ & 13.09 & $3.48 \pm 0.11$ \\
\hline Wing length $(\mathrm{mm} \times 100)$ & $157.12 \pm 0.16$ & 13.93 & $2.38 \pm 0.07$ & $176.64 \pm 0.40$ & 39.93 & $3.58 \pm 0.11$ \\
\hline Bristle number & $9.51 \pm 0.05$ & 1.40 & $12.45 \pm 0.38$ & $8.90 \pm 0.07$ & 2.61 & $18.15 \pm 0.57$ \\
\hline Dev. time (days) & $8.69 \pm 0.01$ & 0.02 & $1.41 \pm 0.04$ & $41.43 \pm 0.10$ & 5.17 & $5.49 \pm 0.17$ \\
\hline Viability & $0.961 \pm 0.003$ & 0.006 & $8.34 \pm 0.26$ & $0.743 \pm 0.008$ & 0.037 & $25.93 \pm 0.84$ \\
\hline
\end{tabular}

Table 2 Mean squares and variance components from three-way nested ANOVAs for five traits at two temperatures. For the morphological traits d.f. is 360 at $25^{\circ} \mathrm{C}$ and 351 at $13^{\circ} \mathrm{C}$

\begin{tabular}{|c|c|c|c|c|c|c|}
\hline \multirow[t]{2}{*}{ Trait } & \multirow[t]{2}{*}{ Source of variation } & \multirow[t]{2}{*}{ d.f. } & \multicolumn{2}{|c|}{$25^{\circ} \mathrm{C}$} & \multicolumn{2}{|c|}{$13^{\circ} \mathrm{C}$} \\
\hline & & & MS & $s^{2} \pm S E$ & MS & $S^{2} \pm S E$ \\
\hline \multirow{4}{*}{ Thorax length ${ }^{\mathrm{a}}$} & Among sires & 29 & $22.49^{*}$ & $0.62 \pm 0.34$ & $38.01^{* *}$ & $1.24 \pm 0.56$ \\
\hline & Among dams & 60 & $11.27^{*}$ & $0.72 \pm 0.38$ & $16.22^{*}$ & $0.91 \pm 0.55$ \\
\hline & Among blocks & 90 & 6.95 & $0.11 \pm 0.38$ & 10.87 & $-0.04 \pm 0.60$ \\
\hline & Among progeny & $360 / 351$ & 6.61 & $6.61 \pm 0.49$ & 11.00 & $11.00 \pm 0.83$ \\
\hline \multirow[t]{4}{*}{ Wing length ${ }^{\mathrm{a}}$} & Among sires & 29 & $67.77^{* * *}$ & $2.55 \pm 0.98$ & $167.74^{* *}$ & $5.48 \pm 2.47$ \\
\hline & Among dams & 60 & $21.82^{* * *}$ & $2.46 \pm 0.68$ & $71.48^{* * *}$ & $7.31 \pm 2.25$ \\
\hline & Among blocks & 90 & 7.09 & $-0.97 \pm 0.43$ & 28.54 & $0.66 \pm 1.55$ \\
\hline & Among progeny & $360 / 351$ & 9.99 & $9.99 \pm 0.74$ & 26.59 & $26.59 \pm 2.00$ \\
\hline \multirow[t]{4}{*}{ Bristle number } & Among sires & 29 & $5.64^{* * *}$ & $0.21 \pm 0.08$ & $13.65^{* * *}$ & $0.59 \pm 0.20$ \\
\hline & Among dams & 60 & $1.80^{* *}$ & $0.13 \pm 0.06$ & $3.19^{*}$ & $0.21 \pm 0.11$ \\
\hline & Among blocks & 90 & 1.04 & $-0.01 \pm 0.06$ & 1.97 & $0.08 \pm 0.11$ \\
\hline & Among progeny & $360 / 351$ & 1.09 & $1.09 \pm 0.08$ & 1.74 & $1.74 \pm 0.13$ \\
\hline \multirow[t]{4}{*}{ Dev. time ${ }^{b}$} & Among sires & 29 & $4.53^{*}$ & $0.11 \pm 0.07$ & 941.20 & $1.49 \pm 16.11$ \\
\hline & Among dams & 60 & 2.48 & $0.06 \pm 0.09$ & $914.25^{*}$ & $61.94 \pm 30.44$ \\
\hline & Among blocks & 90 & $2.10^{* * *}$ & $0.38 \pm 0.11$ & $542.60^{*}$ & $44.08 \pm 28.54$ \\
\hline & Among progeny & 360 & 0.95 & $0.95 \pm 0.07$ & 410.35 & $410.35 \pm 30.50$ \\
\hline \multirow[t]{4}{*}{ Viability $^{c}$} & Among sires & 29 & 7.20 & $0.08 \pm 0.12$ & $20.29^{* *}$ & $0.60 \pm 0.30$ \\
\hline & Among dams & 60 & $5.83^{+}$ & $0.29 \pm 0.20$ & 9.42 & $0.16 \pm 0.35$ \\
\hline & Among blocks & 90 & $4.10^{* * *}$ & $0.62 \pm 0.21$ & $8.47^{* *}$ & $1.08 \pm 0.44$ \\
\hline & Among progeny & 360 & 2.24 & $2.24 \pm 0.17$ & 5.23 & $5.23 \pm 0.39$ \\
\hline
\end{tabular}

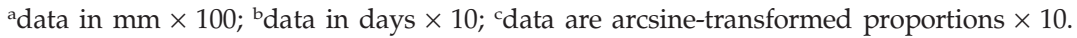

$+P<0.10,{ }^{*} P<0.05,{ }^{* *} P<0.01,{ }^{* * *} P<0.001$.

pleural bristles. It should be noted, however, that for wing length and especially for developmental time, the between-temperature differences were partly explained by scale effects (see Table 1).

Table 3 gives estimates of heritability, coefficient of additive genetic variation and evolvability based on the among-sire and among-dam variances as well as on the sum of the two components (sire + dam). For each trait, $h^{2}$ dam was not significantly higher than $h^{2}$ sire within temperature regimes (a test for the presence of non-additive effects) with the exception of developmental time at $13^{\circ} \mathrm{C}$ (one-tailed $P<0.01$ ). No statistically significant differences were found between stressful and non-stressful environments for the estimates based on the same component of the variance (two-tailed tests, results not shown). The $h^{2}$ sire values tended to be higher for sternopleurals and viability at $13^{\circ} \mathrm{C}$ and for wing length and developmental time at $25^{\circ} \mathrm{C}$. Trends for changes in $h^{2}$ dam were most evident for developmental time and viability (higher at $13^{\circ} \mathrm{C}$ and at $25^{\circ} \mathrm{C}$, respectively). The estimates of $h^{2}$ sire+dam were very similar under stressful and non-stressful conditions for thorax length, developmental time and viability. For wing length and bristle number, the direction of changes in $h^{2}$ sire+dam with temperature coincided with that for $h^{2}$ sire but differences in the absolute values between the two environments were relatively small.

Coefficients of additive genetic variation and evolvabilities for the morphological traits tended to have higher values at $13^{\circ} \mathrm{C}$ regardless of which variance component (sire, dam or sire + dam) they were based on. For the lifehistory traits, $C V_{\mathrm{A}}$ and $I_{\mathrm{A}}$ obtained from the sum of the among-sire and among-dam variances also tended to be higher under stress. However, the sire- and dam-based estimates for these traits did not reveal a consistent pattern of change with temperature. $\mathrm{CV}_{\mathrm{A}}$ and $I_{\mathrm{A}}$ were generally higher at $13^{\circ} \mathrm{C}$ when estimated using the among-sire 
Table 3 Heritabilities, coefficients of additive variation and evolvabilities for five traits at two temperatures based on different variance components

\begin{tabular}{|c|c|c|c|c|c|c|c|}
\hline \multirow[t]{2}{*}{ Trait } & \multirow[t]{2}{*}{ Variance component } & \multicolumn{3}{|c|}{$25^{\circ} \mathrm{C}$} & \multicolumn{3}{|c|}{$13^{\circ} \mathrm{C}$} \\
\hline & & $h^{2} \pm S E$ & $C V_{\mathrm{A}}$ & $I_{\mathrm{A}} \times 10^{4}$ & $h^{2} \pm S E$ & $C V_{\mathrm{A}}$ & $I_{\mathrm{A}} \times 10^{4}$ \\
\hline \multirow{3}{*}{ Thorax length } & Sire & $0.309 \pm 0.176$ & 0.73 & 0.54 & $0.379 \pm 0.143$ & 1.07 & 1.15 \\
\hline & Dam & $0.357 \pm 0.167$ & 0.79 & 0.62 & $0.278 \pm 0.160$ & 0.92 & 0.84 \\
\hline & Sire + dam & $0.333 \pm 0.083$ & 1.07 & 1.15 & $0.328 \pm 0.078$ & 1.41 & 1.99 \\
\hline \multirow[t]{3}{*}{ Wing length } & Sire & $0.728 \pm 0.189$ & 1.02 & 1.03 & $0.547 \pm 0.179$ & 1.33 & 1.76 \\
\hline & Dam & $0.700 \pm 0.197$ & 1.00 & 1.00 & $0.731 \pm 0.202$ & 1.53 & 2.34 \\
\hline & Sire + dam & $0.714 \pm 0.098$ & 1.42 & 2.03 & $0.639 \pm 0.103$ & 2.02 & 4.10 \\
\hline \multirow[t]{3}{*}{ Bristle number } & Sire & $0.603 \pm 0.205$ & 4.85 & 23.53 & $0.908 \pm 0.249$ & 8.67 & 75.16 \\
\hline & Dam & $0.358 \pm 0.165$ & 3.73 & 13.94 & $0.318 \pm 0.164$ & 5.13 & 26.30 \\
\hline & Sire + dam & $0.480 \pm 0.079$ & 6.12 & 35.68 & $0.613 \pm 0.073$ & 10.07 & 90.56 \\
\hline \multirow[t]{3}{*}{ Dev. time } & Sire & $0.302 \pm 0.158$ & 0.39 & 0.15 & $0.012 \pm 0.075$ & 0.29 & 0.09 \\
\hline & Dam & $0.168 \pm 0.147$ & 0.29 & 0.08 & $0.478 \pm 0.176$ & 1.90 & 3.61 \\
\hline & Sire + dam & $0.235 \pm 0.071$ & 0.48 & 0.23 & $0.245 \pm 0.095$ & 1.92 & 3.70 \\
\hline \multirow[t]{3}{*}{ Viability } & Sire & $0.095 \pm 0.124$ & 1.88 & 3.54 & $0.342 \pm 0.146$ & 7.17 & 51.39 \\
\hline & Dam & $0.356 \pm 0.165$ & 3.64 & 13.27 & $0.090 \pm 0.139$ & 3.67 & 17.89 \\
\hline & Sire + dam & $0.225 \pm 0.086$ & 4.10 & 16.81 & $0.216 \pm 0.065$ & 8.06 & 64.89 \\
\hline
\end{tabular}

variances for viability and among-dam variances for developmental time, whereas dam-based estimates for the first trait and sire-based estimates for the second trait seemed not to be affected by temperature.

\section{Discussion}

Rearing flies under low-temperature stress had a strong effect on phenotypic variation. All phenotypic variances and coefficients of variation were significantly higher at $13^{\circ} \mathrm{C}$ than at $25^{\circ} \mathrm{C}$. The same pattern was found for environmental variation estimated via among-progeny variance. The estimates of genetic variation did not reveal statistically significant differences between the two environments. However, the pattern of their values, considered in the context of results from other experiments on $D$. melanogaster, indicates the possibility of changes in genetic variation at the stressful temperature.

The effect of extreme low temperature on the same set of traits has been recently investigated by Imasheva et al (1998), who analysed quantitative genetic variation using the isofemale line technique. In their work, among-line variances for all five traits tended to be higher at $12^{\circ} \mathrm{C}$ than at $25^{\circ} \mathrm{C}$. In other isofemale line studies, in which thorax and wing length were examined (de Moed et al, 1997; Karan et al, 1999; Imasheva et al, 2000), a clear-cut trend for an increase in the among-line variances at low temperatures was also demonstrated. However, in the experiment with parent-offspring comparisons Sgrò and Hoffmann (1998) did not find a difference between $14^{\circ} \mathrm{C}$ and $28^{\circ} \mathrm{C}$ (the latter seems to be a suboptimal rather than a stressful temperature; see for example David et al, 1983) in the genetic variance of wing length, while for developmental time this variance was significantly lower at $14^{\circ} \mathrm{C}$.

In our study, we considered three variances that reflect genetic variation. The among-sire (parental half-sib) variance is similar to the genetic variance from the parentoffspring regression analysis and includes, in addition to the additive component, a small proportion of non-addi- tive (epistatic) variance (Falconer and Mackay, 1996). The among-dam variance contains the same genetic components as the variance among isofemale lines (additive, dominance, epistatic and the variance due to maternal effects) but with a relatively smaller proportion of the additive variance. The sum of the among-sire and amongdam variances gives the full-sib variance, which is equivalent to the variance among isofemale lines. We found that this sum tended to be higher at the low stressful temperature for all traits, which is in good agreement with the behaviour of among-line variances in the isofemale line studies by de Moed et al (1997), Imasheva et al (1998, 2000) and Karan et al (1999). Moreover, the trend for changes in the among-sire variances for thorax length, wing length, bristle number and viability also coincides with that for the among-line variances reported by the above-mentioned authors. These facts indicate that for these traits the estimates of the additive variance across temperatures based on isofemale line data are quite reasonable and the level of additive genetic variation is likely to increase under low-temperature stress. However, for developmental time, the trend for change in the among-sire variance is consistent with a decrease in genetic variance in the parent-offspring comparisons by Sgrò and Hoffmann (1998) and this warns against using the isofemale line technique to investigate changes in the additive genetic variance in this trait. Our analysis revealed a trend for an increase in the among-dam variance of developmental time in the stressful environment that may be related to the enhanced level of non-additive genetic variation.

In the studies of de Moed et al (1997), Imasheva et al $(1998,2000)$ and Sgrò and Hoffmann (1998), heritabilities showed the same trends for changes across temperatures as the genetic variances on which they were based. However, in the present experiment a more complicated pattern was found. The estimates based on the among-sire and among-dam variances were more variable than those obtained from the sum of the two components (ie, fullsib estimates) that reflected opposite trends of changes for 
among-sire and among-dam variances within some traits. For thorax length, developmental time and viability, the full-sib estimates seemed to be equal and only for wing length and bristle number did they show a weak trend for changes in the same direction as the sire-based heritabilities (decreased for wing length and increased for bristle number). The sire-based heritabilities, which are theoretically the least biased estimates of the narrow heritability in the half-sib design (Falconer and Mackay, 1996), tended to be higher at the stressful temperature for bristle number and viability and lower for wing length and developmental time, while for thorax length they appeared not to be affected by temperature. Such different trends can be explained by the fact that an increase in environmental variance (which enters the denominator in the expression for heritability) under stress was relatively greater for some traits than for others. Thus, our data illustrates a common situation where evolutionary implications cannot easily be drawn from changes in heritability estimates alone.

To overcome this difficulty, Houle (1992) proposed other measures of the ability of a population to respond to selection, the coefficient of genetic variation $\left(\mathrm{CV}_{\mathrm{A}}\right)$ and evolvability $\left(I_{\mathrm{A}}\right)$. These indices may also be preferable to the additive genetic variance, as they are not affected by trait size. We considered $\mathrm{CV}_{\mathrm{A}}$ and $I_{\mathrm{A}}$ based on sire, dam and sire + dam components of genetic variance. Regrettably, standard errors for these estimates are not known and it was not possible to carry out statistical comparisons between environments. Generally, changes in different $\mathrm{CV}_{\mathrm{A}}$ and $I_{\mathrm{A}}$ estimates showed the same trends as the variance components used for their calculation with the exception of two cases: the among-sire variability for developmental time and the among-dam variability for viability seemed to be not influenced by temperature when estimated with $\mathrm{CV}_{\mathrm{A}}$ and $I_{\mathrm{A}}$. Thus, we can say that, under low temperature stress, the level of additive genetic variation tends to be higher for thorax length, wing length, bristle number and viability, while for developmental time an increase in nonadditive genetic variation may occur.

The same trends in behaviour of genetic variation in a stressful environment have been recently found in the same $D$. melanogaster stock for the above-listed traits at $32^{\circ} \mathrm{C}$ and at $25^{\circ} \mathrm{C}$ (Bubliy and Loeschcke, 2001). A similar situation was also reported in the study of Imasheva et al (1998), in which among-line variances for the same traits, except developmental time, tended to increase both at $12^{\circ} \mathrm{C}$ and $31^{\circ} \mathrm{C}$ compared with $25^{\circ} \mathrm{C}$. In addition, the among-line variances and coefficients of genetic variation of morphological traits analysed in D. melanogaster by Karan et al (1999) and Imasheva et al (2000) tended to be higher at temperature extremes. All these data suggest that high- and low-temperature stress may affect genetic variation of a trait in the same way and for most traits considered here the effect of stress is in agreement with the hypothesis (Hoffmann and Parsons, 1991) of an increase in the level of additive genetic variation in stressful environments.

The difference between developmental time and other traits in response of their genetic variation to the temperature stress can be viewed as an indication of trait-specific effects of extreme temperatures. On the other hand, there is evidence that effects of some stressors on the examined morphological traits may not be consistent with the aforementioned hypothesis. It has been shown (Hoffmann and
Schiffer, 1998; Bubliy et al, 2000, 2001) that nutritional stress in D. melanogaster did not change or even decreased additive genetic variation for wing length and sternopleural bristle number, while for thorax length this variation displayed a clear trend to be higher under poor nutrition. These facts warn against generalizations about behaviour of genetic variation based on results of testing using single traits and stressors.

\section{Acknowledgements}

We thank Andrew Davis for providing flies for the experiment, Doth Andersen and Trine Gammelgaard for technical assistance and Stuart (JSF) Barker for helpful comments on the manuscript. The research was supported by the Danish Natural Science Research Council (grant no. 00031) and the ESF Scientific Programme on Theoretical Biology of Adaptation (travel grant to OAB). VL also thanks the Institute for Advanced Study and the Center for Environmental Stress and Adaptation Research at La Trobe University, where he was a visiting research fellow when working at the manuscript, for their hospitality.

\section{References}

Barker JSF, Krebs RA (1995). Genetic variation and plasticity of thorax length and wing length in Drosophila aldrichi and $D$. buzzatii. J Evol Biol 8: 689-709.

Becker WA (1992). Manual of Quantitative Genetics, 5th edn. Academic Enterprises: Pullman, Washington.

Bubliy OA, Imasheva AG, Loeschcke V (2000). Half-sib analysis of three morphological traits in Drosophila melanogaster under poor nutrition. Hereditas 133: 59-63.

Bubliy OA, Loeschcke V (2001). High stressful temperature and genetic variation of five quantitative traits in Drosophila melanogaster. Genetica 110: 79-85.

Bubliy OA, Loeschcke V, Imasheva AG (2001). Genetic variation of morphological traits in Drosophila melanogaster under poor nutrition: isofemale lines and offspring-parent regression. Heredity 86: 363-369.

David JR, Allemand R, van Herrewege J, Cohet Y (1983). Ecophysiology: abiotic factors. In: Ashburner M, Carson HL, Thompson JN (eds) Genetics and Biology of Drosophila, Academic Press: London. Vol 3d, pp 105-170.

David JR, Moreteau B, Gauthier JP, Petavy G, Stockel A, Imasheva AG (1994). Reaction norms of size characters in relation to growth temperature in Drosophila melanogaster: an isofemale lines analysis. Genet Sel Evol 26: 229-251.

de Moed GH, de Jong G, Scharloo W (1997). Environmental effects on body size variation in Drosophila melanogaster and its cellular basis. Genet Res 70: 35-43.

Falconer DS, Mackay TFC (1996). Introduction to Quantitative Genetics, 4th edn. Longman: Essex.

Gebhardt MD, Stearns SC (1992). Phenotypic plasticity for lifehistory traits in Drosophila melanogaster. III. Effect of the environment on genetic parameters. Genet Res 60: 87-101.

Hoffmann AA, Parsons PA (1988). The analysis of quantitative variation in natural populations with isofemale strains. Génét Sél Evol 20: 87-98.

Hoffmann AA, Parsons PA (1991). Evolutionary Genetics and Environmental Stress. Oxford University Press: Oxford, New York.

Hoffmann AA, Schiffer M (1998). Changes in the heritability of five morphological traits under combined environmental stresses in Drosophila melanogaster. Evolution 52: 1207-1212.

Houle D (1992). Comparing evolvability and variability of quantitative traits. Genetics 130: 195-204.

Imasheva AG, Bosenko DV, Bubli OA (1999). Variation in morphological traits of Drosophila melanogaster (fruit fly) under nutritional stress. Heredity 82: 187-192. 
Imasheva AG, Loeschcke V, Zhivotovsky LA, Lazebny OE (1998). Stress temperatures and quantitative variation in Drosophila melanogaster. Heredity 81: 246-253.

Imasheva AG, Moreteau B, David JR (2000). Growth temperature and genetic variability of wing dimensions in Drosophila: opposite trends in two sibling species. Genet Res 76: 237-247.

Karan D, Morin JP, Gravot E, Moreteau B, David JR (1999). Body size reaction norms in Drosophila melanogaster: temporal stability and genetic architecture in a natural population. Genet Sel Evol 31: 491-508.
Loeschcke V, Bundgaard J, Barker JSF (1999). Reaction norms across and genetic parameters at different temperatures for thorax and wing size traits in Drosophila aldrichi and D. buzzatii. J Evol Biol 12: 605-623.

Sgrò CM, Hoffmann AA (1998). Effects of temperature extremes on genetic variances for life history traits in Drosophila melanogaster as determined from parent-offspring comparisons. I Evol Biol 11: 1-20.

Sokal RR, Rohlf FJ (1995). Biometry, 3rd edn. W. H. Freeman and Co: New York. 